\title{
Performance Testing for VoIP Emergency Services: a Case Study of the EMYNOS Platform and a Reflection on potential Blockchain Utilisation for NG112 Emergency Communication
}

\author{
Budankailu Sameer Kumar Subudhia, Faruk Catal ${ }^{a}$, Nikolay Tcholtchev ${ }^{\mathrm{a}}$, Kin Tsun Chiu ${ }^{\mathrm{a}}$, Yacine \\ Rebahi $^{\mathrm{a}}$, Michell Boerger ${ }^{\mathrm{a}}$ and Philipp Lämmel ${ }^{\mathrm{a}}$
}

${ }^{a}$ Fraunhofer FOKUS, Kaiserin-Augusta-Allee 31, Berlin, 10589, Germany

\begin{abstract}
VoIP-based emergency communication is a promising approach to improving the safety of citizens worldwide. The transition required in this scope includes substituting the legacy PSTN/SS7 based emergency call system by Next Generation IP based components for call establishment and control. Thereby, SIP is used as a session control protocol and RTP as the means to transfer emergency data between the caller and the corresponding Public Safety Access Point (PSAP). The emergency data is not only restricted to voice communication but can cover a rich variety of data, which can be acquired by different means (including the end-user devices) and transmitted over IP. This includes video, geopositioning data, voice, Real-Time Text, and sensor data in line with emerging IoT architectures and approaches. A vital aspect in this scope is given by the performance of the underlying network, including its capability to establish calls in emergencies and to transfer the data required for serving the situation. Therefore, in this paper, we evaluate the computational performance of the most recent VoIP emergency system implementation, which was developed by the H2020-EMYNOS project as a realisation of the EENA NG112 Long Term Definition (LTD) vision. We perform a series of trials and evaluate the performance of the EMYNOS system in a multi-party lab environment established during the project. We evaluate the time needed to perform basic emergency call operations over IP, whilst in parallel generating Internet type of background traffic. Correspondingly, we worked out a methodology and implemented it in our testbed, both of which are presented in the current paper. The obtained numerical results lead to the conclusion that SIP-based emergency services stand a good chance to replace legacy systems when it comes to their performance. Additionally, we also provide a perspective on how the blockchain technology could potentially be put to use to enhance the quality of the next-generation emergency services. We propose the utilisation of blockchain technology for tracking emergency calls and enabling efficient recognition of fraud calls, which is a critical aspect for PSAP providers concerning the potential denial of service attacks. In this context, we provide evaluations and numerical results based on a private Ethereum based blockchain playground running at the premises of Fraunhofer FOKUS.
\end{abstract}

Keywords: performance testing; SIP; VoIP; NG112; PSAP; NGN; background traffic; blockchain; fraud-detection

\section{Introduction}

The nExt generation eMergencY commuNicatiOnS (EMYNOS) project [1] is a European-funded project aiming to address the current emergency systems limitations, particularly in terms of media and location. This project, that ended February 2018, was discussed in details in our paper [2] and produced a platform for enabling European citizens to make IP-based emergency calls (to the police, the ambulance and the fire brigade). Indeed, the output of EMYNOS was a Next
Generation emergency call full chain, starting from the caller, going through the network (in particular, the ESInet [3]), and terminating at the emergency call taker client. Various functionalities such as emergency call identification, caller/device location configuration, routing to the appropriate emergency call centre, location information visualisation, sensor data transmission, and protection against false calls were addressed. The EMYNOS platform intersects the NG112 architecture, described in the EENA NG112 LTD document [3], and implements the related functionalities according to the project consortium needs and requirements.

${ }^{*}$ Corresponding author. Tel.: +49 30 3463-7175

E-mail: nikolay.tcholtchev@fokus.fraunhofer.de

C 2020 International Association for Sharing Knowledge and Sustainability.

DOI: 10.5383/JUSPN.12.01.001 


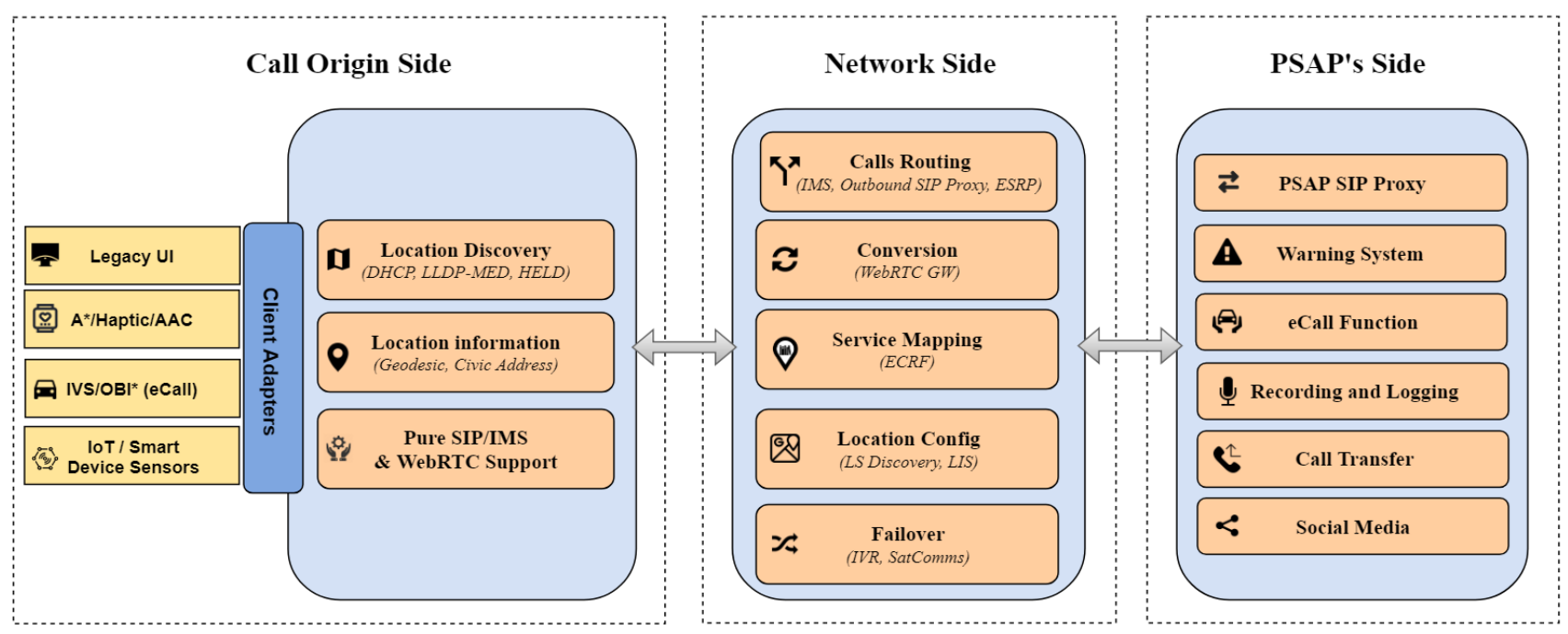

\section{Fig. 1: EMYNOS high-level Architecture}

To evaluate the EMYNOS framework, several experiments were conducted at various sites. This includes the development testbed established at the TEIC premises in Crete, as well as the one at Fraunhofer FOKUS in Berlin [4]. The undertaken testing activities were initially discussed in [2]. It is worth mentioning that in the test cases performed in [2], background traffic was not taken into account as the testbed was not fully operational. In the current paper, we present the final results of the performance testing procedures within the EMYNOS project, including various advanced scenarios and the evaluation with supplementary background traffic. The traffic was generated based on open source tools, which are widely used in the community, and a corresponding methodology and belonging test components were developed and scripted - once for the generation and measurement of the SIP traffic and once for the background data. Thereby, the innovation of this paper is given by exemplifying on how NGN based emergency platforms can be tested regarding their performance and their operational limits. Since the EMYNOS platform constitutes a pioneer in this type of platforms, the VoIP features of NGN 112 are systematically examined in an innovative setup which is rarely available to the public (especially as open-source). Furthermore, this paper deals with the integration of NG112 with emerging blockchain platforms and their utilisation for fraud detection. In this scope, we provide indicative performance measurements showing the feasibility and applicability of blockchain for denial-of-service (DoS) call detection on the PSAP - functionality which is of crucial importance for a critical infrastructure supporting emergency communications. Thereby, the interplay between an emergency communication infrastructure and immutable storage is of paramount importance for guaranteeing the proper functioning of an NG 112 VoIP emergency service, provided that a large number of fraudulent calls can lead to reduced availability of such critical infrastructure. Hence, we examine different performance-related aspects, to approach the topic also from this perspective.

The structure of this paper is as follows: Section 2 discusses the current state of the art. Section 3 presents an overview of the EMYNOS platform. We describe the testbed in section 4 . In section 5 , we discuss the setup followed by the testing methodology and numerical results in Section 6. In Section 7, we propose a novel idea to fight hoax emergency calls using blockchain and conclude the paper with section 8 .

\section{Related Work}

The utilisation of VoIP and SIP for emergency communication has been a topic for intensive research during the past decades with [6], [7], [8], [9] and [10] being some of the research works in very recent years. Besides a European mandate for emergency communication M.493 [11] has been established by ETSI and the European Commission, to regulate the setup of VoIP emergency services' architectures according to European law and jurisdiction. It is also worth mentioning that NG911 (perceived as NG112 in Europe) was initially pushed for in the USA thereby establishing standards for facilitating the adoption of this promising technology [12]. Furthermore, several European projects have investigated emergency call architectures over IP with [13], [15], [16] and [17] being some of the notable examples in the area. It is also important mentioning that the topic of eCall [19], i.e. the automated triggering of emergency calls, has led to various standards utilising the traditional SS7 [18] control stack. Especially in the automotive domain, eCall is close to deployment being pushed by corresponding European regulations.

The testing work related to Next Generation emergency services and following the EENA NG112 LTD vision is very scarce. Two main activities were undertaken in 2016 and 2017 under the umbrella of the ETSI NG112 plugtests [5]. These activities were focusing more on interoperability. To our knowledge, performance testing in the context of Next Generation emergency services was presented first by the authors of this paper in [2] where no background traffic was considered.

An attempt at fraud detection and prevention related to IP Multimedia Subsystem (IMS) Networks have been made in [23] and [24]. A survey report on the exploitation of VoIP networks and available solutions has been published in [25]. And lastly, a patent in the USA exists for the "Processing of emergency calls in wireless communications system with fraud protection." [28].

\section{EMYNOS Platform}

The EMYNOS platform has been developed to enable, in particular, smarter communication services in case of 


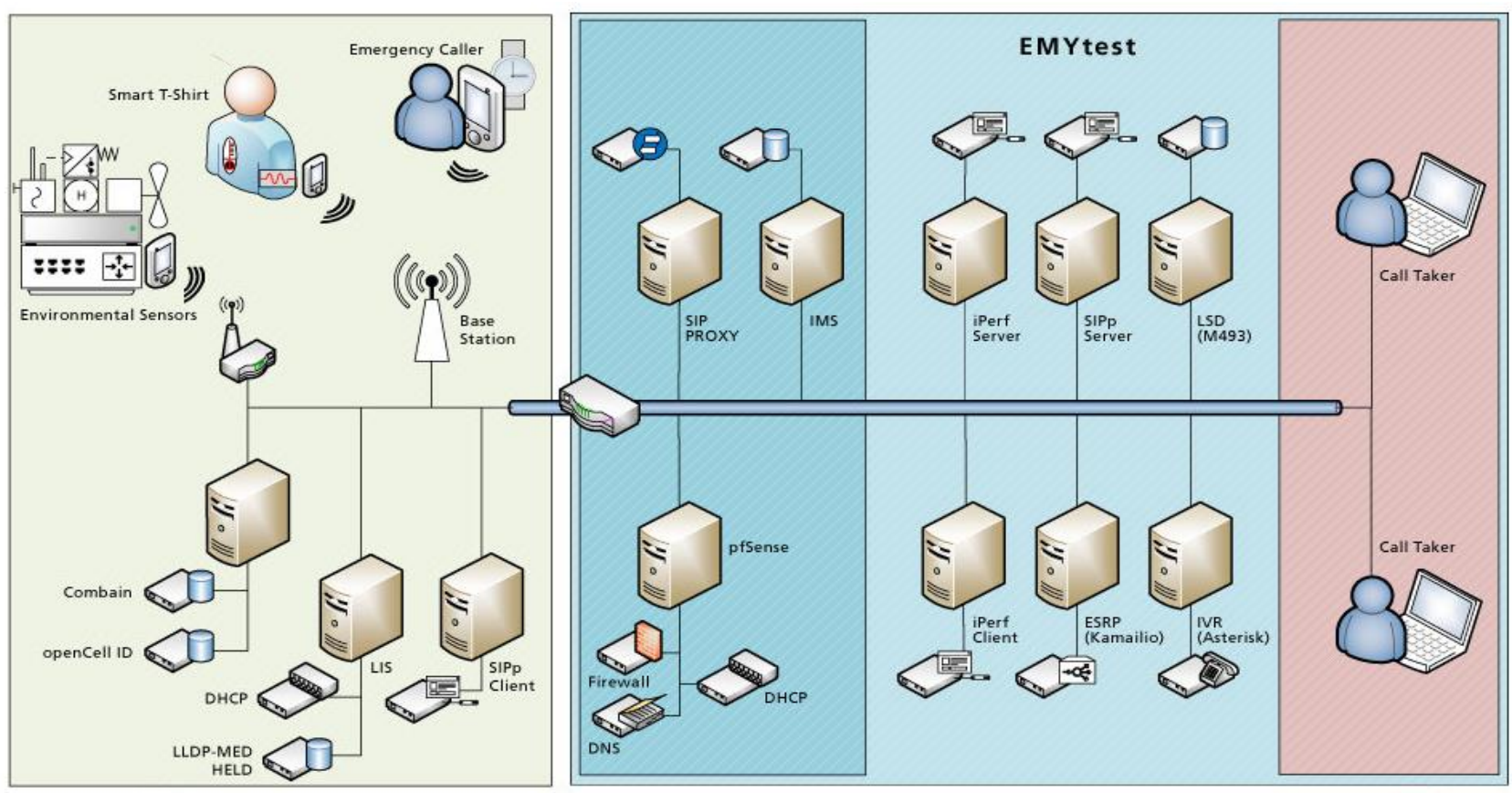

Fig. 2 EMYNOS Testbed in Fraunhofer FOKUS premises

emergencies. The involved actors can be classified into two categories; namely the Caller and the Callee. Fig. 1 provides an overview of the complete platform. More information on the actors and the platform are mentioned below*.

The Callee, in the EMYNOS framework, can be an agency that provides assistance services to handle any emergency the Caller is in. Callees may include generic Public Safety Answering Point (PSAP) call takers or even a purposespecific agency such as the police, the ambulance or the firefighting services. The Caller in EMYNOS can be any person placing an emergency call or even a device that implements a SIP or WebRTC enabled client. The generated calls can be initiated either automatically or manually. Devices such as smartwatches, haptic-sensor or any other (health-) sensor (e.g. smart t-shirt) based devices can trigger an automatic emergency call. A person can also initiate an emergency call manually. All these calls are generated from a Voice over Internet Protocol (VoIP) client that internally makes use of the Session Initiation Protocol (SIP) and Web Real-Time Communication (WebRTC) protocols. Sometimes, these caller devices may also send their location information to assist the platform in smartly resolving the closest relevant emergency response service. The payload information for resolving the location-based service in the calls are supported on the above devices using Dynamic Host Configuration Protocol (DHCP), Link Layer Discovery Protocol - Media Endpoint Discovery (LLDP-MED) or HTTP - Enabled Location Delivery (HELD) protocols. The network components of the EMYNOS framework consist of either an outbound SIP proxy or an IP Multimedia Subsystem (IMS) that interacts with a HELD based Location Server to determine the location-based service, then to an Emergency Services Routing Proxy (ESRP) and an Emergency Call Routing Function (ECRF) that help in final routing of the call to the appropriate emergency call center, based on the type of the emergency call and the caller location.

\footnotetext{
* The authors refer to [2] for a more detailed overview of standards for NG emergency communication architectures.
}
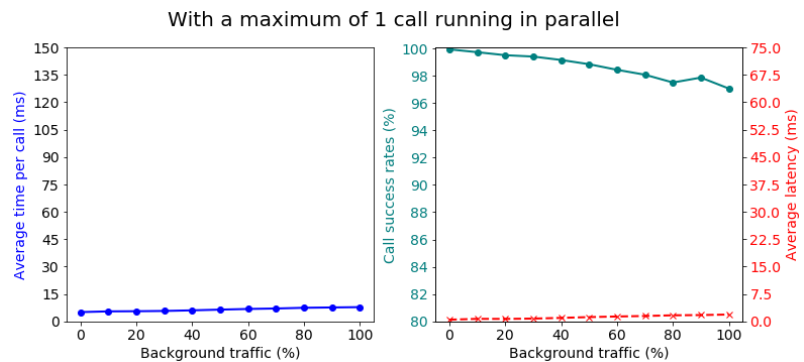

Fig. 3 SIP call establishment with maximum 1 call in parallel

\section{Testbed}

A testbed implementing all the intricate details stated above has been set up for development, evaluation and demonstration purposes at the Technological Educational Institute of Crete, Greece (TEIC) premises on which all the project partners have been primarily working. The same setup (see Fig. 2) has also been replicated locally at the premises of Fraunhofer Institute for Open Communication Systems (FOKUS), on which the performance tests were run and the results are presented here.

As depicted in Fig. 2, the testbed components consist of a SIP Proxy (Kamailio based), an ESRP (Kamailio based), a couple of iPerf instances (Client \& Server nodes generating TCP and UDP traffic in parallel) and a couple of SIPp instances (Caller \& Callee modes). The iPerf instances are used to generate and mimic the background traffic in the network in a controlled manner. SIPp is used to mimic the emergency callers and callees. The Asterisk - acting as PBX (Private Branch Exchange) - is required to automatically distribute and delegate the high volume of incoming calls during the emergencies among the various agents available at the PSAP. And in certain cases where all the callees are busy, the PBX component also doubles as an Interactive Voice Response 

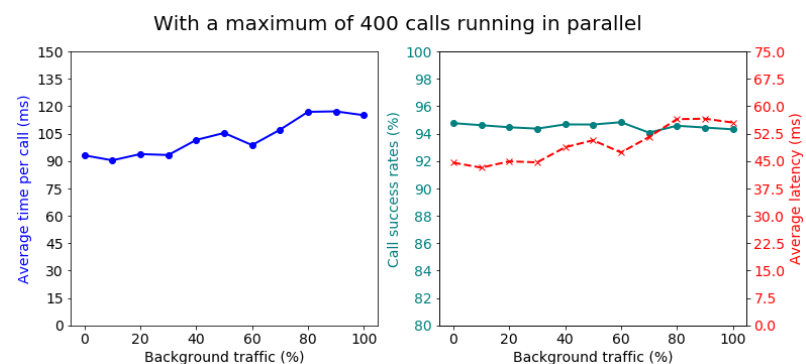

Fig. 5 SIP call establishment with maximum 400 calls in parallel

(IVR) system to respond the callers with a relevant prerecorded message.

\section{Experimental Setup}

The envisioned test was intended at determining the performance of the primary components of the EMYNOS platform (including the SIP proxy and eventually the ESRP) and its ability to handle the traffic under distress, as would be the case whenever an emergency scenario occurs. The metrics were selected based on different discussions within the EMYNOS project, to provide a reasonable indication regarding the real-world applicability of an NG 112 VoIP system. To enable the testing, other supportive tools were used, namely SIPp and iPerf. Two SIPp instances were used to emulate the emergency calls in the roles of the emergency caller and the emergency call taker. Also, to determine the bandwidth of the network and emulate the background traffic, two instances of iPerf were used. All the test components in the testbed were setup on separate Virtual Machine (VM) instances to run separately and independent of each other. The VMs host Ubuntu Linux 16.04 on a virtualised environment consisting of Intel Xeon CPU E5420 @ 2.50 GHz, 2.0 GB RAM and 9.4 GB HDD. A major part of the performance testing procedure was automated using shell scripts. The shell scripts were developed to be dynamic and parameterised, and use configuration files specific to each available testbed.

This setup was also used to evaluate various tools under consideration. The tools used for the tests, apart from the various components constituting the EMYNOS platform, could be fit into two categories: 1) The Actor Emulators - as stated earlier, we use SIPp to emulate the actors in this case, namely the Caller and the Callee. 2) Network traffic Simulators - again as previously stated, we use iPerf to simulate and saturate the network bandwidth with background traffic in a controlled manner.

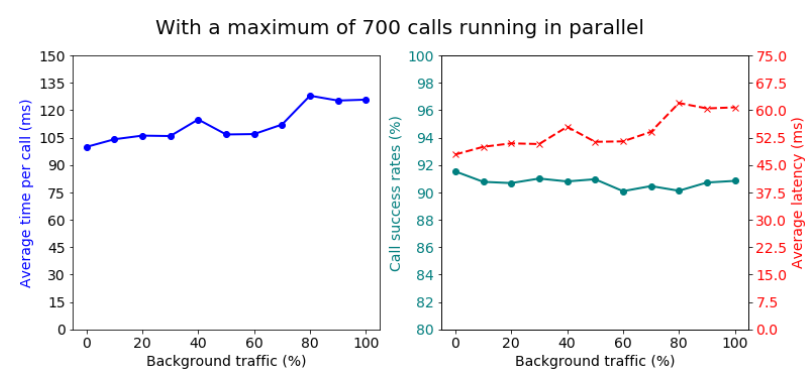

Fig. 6 SIP call establishment with maximum 700 calls in parallel

\section{Methodology \& Numerical Results}

SIPp, the emulator for the actors involved in an emergency call, lets us define custom call scenarios to mimic various user actions in an XML file called the scenario file. These scenario files can also dynamically accept various values from an injection file. We use the injection file methodology to inject the caller names, authentication and various other details dynamically from a list that has one thousand callers defined in it.

With Average Time per call, Average Latency and Call Success Rates as the required metrics in mind, we designed an end-to-end call scenario. In the custom scenario we fabricated, the Caller places an emergency call to the Callee over the EMYNOS platform. As soon as the Callee responds to the call successfully, the call is ended by the Caller. Since all the calls take place over the internet supporting infrastructure, the communication protocol put to use is SIP, as was mentioned earlier. The next paragraph explains the scenario in technical terms.

$S I P p$ emulates the Caller scenario as follows. It begins with sending out a REGISTER request to the ESRP. In case the request requires authentication, the same REGISTER request is resent now along with the authentication details. Once the Caller is successfully registered with the ESRP, it sends out an INVITE message to the Callee with priority set to emergency,

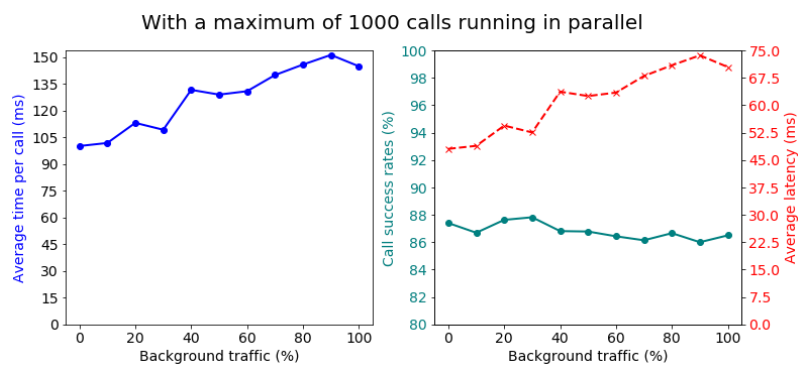

Fig. 4 SIP call establishment with maximum 1000 calls in parallel

geo-location and other necessary accompanying data payload in the Session Description Protocol (SDP). The call priority is set using the Priority field in the INVITE message as "Priority: emergency". The Caller then expects a 100 TRYING followed by a $180 R I N G$ and a final $200 O K$ that confirms that the Callee has answered the call. The Caller then sends out an $A C K$ message immediately followed by a $B Y E$ to terminate the call. Once the Caller receives the final $200 \mathrm{OK}$, corresponding to the $B Y E$, from the Callee the emergency call scenario terminates. Contrary to the Caller, the Callee emulation takes place slightly differently in SIPp. The Callee registers itself with the ESRP as a service just once throughout the complete testing procedure. This is required as the Callee needs to be available online all the time to accept an emergency call. Once the Callee is registered with the ESRP, SIPp now runs the Callee response scenario. The Callee response scenario begins with an expected INVITE request. As soon as it receives an INVITE request, it responds with a 180 RINGING immediately followed by a 200 $O K$, which informs the Caller that the Callee has now accepted the call. Then, corresponding to the Caller scenario definition, the Callee now expects to receive an $A C K$ message immediately followed by a $B Y E$ message. As soon as the Callee receives a $B Y E$ message, it acknowledges the BYE message with a $200 \mathrm{OK}$ message and finally terminates the call from its perspective. 
With the understanding of how the Caller and the Callee supported bandwidth was determined, the test scenarios were

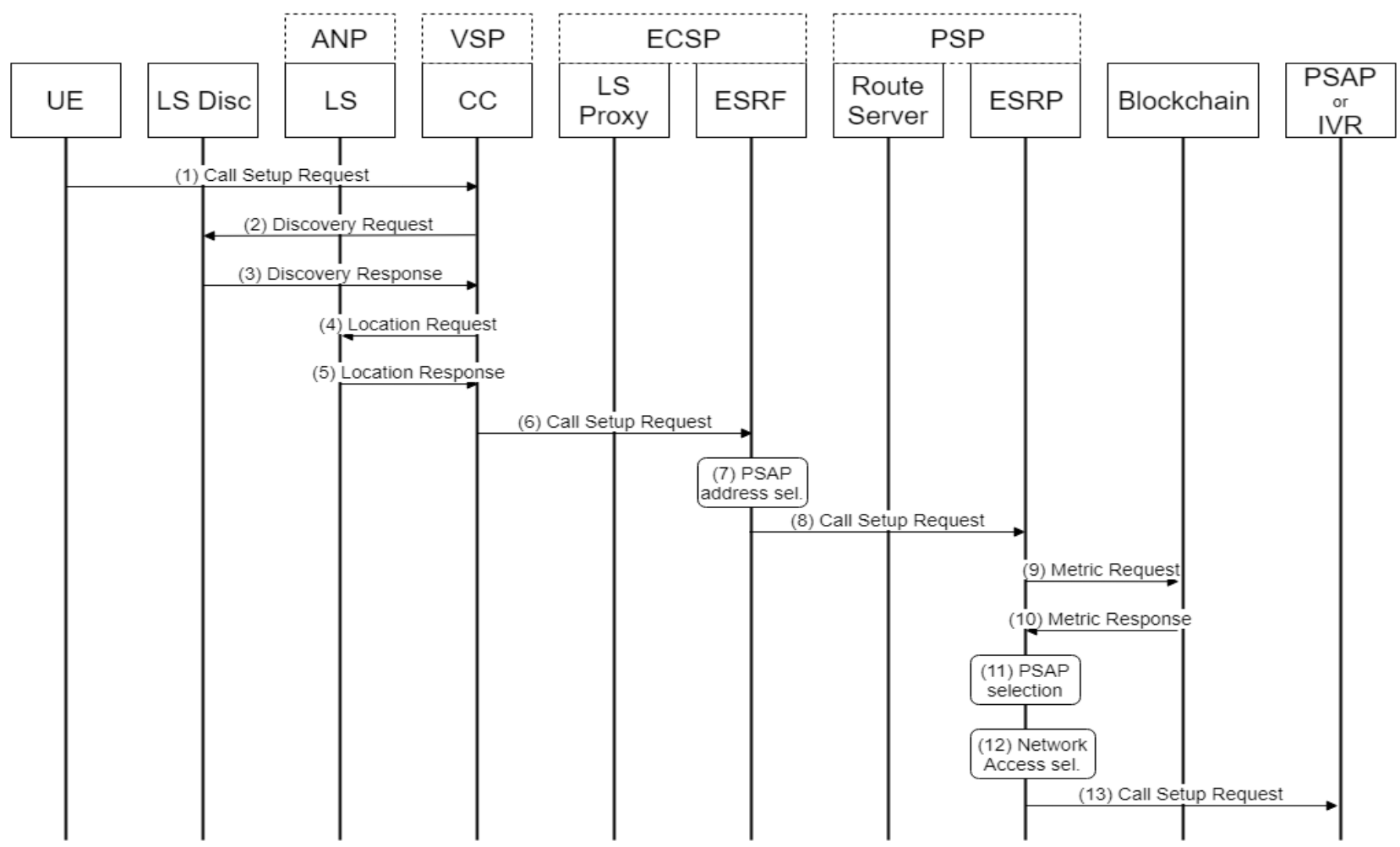

Fig. 7 Sequence diagram for proposed solution for an incoming emergency call

response scenarios have been implemented, let us now explain what timing metrics are captured and how are they used to derive other details. In the Caller scenario definition, we start the timer when the first INVITE message is sent out to the Callee. The timer ends once the call is successfully established and the Caller ends the call with the sending of a $B Y E$ message. Let us refer this as ResTimeCaller. A similar timer is captured on the Callee response scenario. This timer starts once the Callee receives an INVITE request from the Caller and terminates with the sending of a $200 \mathrm{OK}$ message which is sent out in response to the INVITE. Let us refer this as ResTimeCallee. The above call scenario was run multiple times to simulate various kinds of stress level scenarios. Thereby, the goal is to push the available emergency communication platform to the extreme and show how its limits can be examined. The two parameters that were controlled and varied to create the scenarios are:

A maximum number of calls in parallel: We ran the tests by controlling the maximum number of calls that are allowed to be established at any point in time during the test cycle ${ }^{\dagger}$. We ran the tests by executing the calls first sequentially; the subsequent runs allowed 100 calls to existing simultaneously. With every run, the number of calls allowed to run in parallel was increased by 100 until the cap reached 1000 i.e. the values used for the tests were 1, 100, 200, 300, 400...1000.

Level of background traffic: For every setting above, the communication channel was saturated with background traffic to observe its effect on the captured metrics. The maximum bandwidth supported by the SUT (System Under Test) network was determined by using the iPerf tool. Once the maximum

\footnotetext{
$\dagger$ The possible statistical variance due to the Internet segment between the end-users' devices and the EMYtest is taken care of by starting a large number of calls within which trial and obtaining the average value of the established performance indicators.
}

executed, beginning with absolutely no background traffic and then increasing the traffic by $10 \%$ for every subsequent test run.

By combining the two factors explained above, we executed exactly 121 test runs, one for each possible scenario. Each test run was executed with 10000 calls. Thus, for the complete test set, we simulated exactly 12.1 million calls. For every scenario that we ran, the average of the ResTimeCaller and the average of the ResTimeCallee was calculated but only for those calls that completed executing successfully. Let us refer these values as avgResTimeCaller and avgResTimeCallee respectively.

In this paper, we present results from a few selected cases. The results are presented as graphical plots. The graphs plot all the three metrics mentioned earlier (i.e. Average Time per call, Average Latency and Call Success Rates). We define these metrics as:

Each result set presented here is segregated by the maximum number of calls that were allowed to simulate in parallel. All three metrics are plotted against the background traffic. Every image consists of two graphs. The first graph plots the Average Time Taken per Call in milliseconds. The second graph plots the Call Success Rate in percentage on the left and the Average Latency in milliseconds on the right. The Call Success Rate is plotted with the solid line and the Average Latency with the dashed line. The results with the corresponding graphs are plotted in Fig. 3 through Fig. 5 respectively.

The obtained results illustrate the limits of the opensource EMYtest platform for emergency communications within the described setup. Thereby, our goal was mainly to demonstrate and show a methodology regarding how such performance parameters can be measured for innovative scenarios in a VoIP based emergency context. In a productiongrade environment, a series of improvements and hardware 
upgrades would lead to improved performance within the provided communication requirements.

\section{Integrating Blockchain to prevent Fraud Calls}

Emergency services are set up to help people and provide immediate assistance to ensure the safety of the citizens in their times of trouble. When necessary, the PSAP is also required to dispatch the emergency services such as the police, the firefighters, the ambulance or the rescue services. However, at times some people turn antagonist and make fraudulent calls for reasons known and unknown. Such activities at the times of emergency may raise hindrance in providing support services to the people who are in actual need and might lead to tragedies. A report [20], published by the European Emergency Number Association (EENA), states that in certain regions of the EU that as much as about $99 \%$ of the calls made to the emergency services turned out to be either false or a hoax while the hoax calls amounted up to $19 \%$ of the total call count. Another article [21], from KOVA Corp. - a vendor for call recording solutions in the USA for the public safety community, claims $90 \%$ of the 911 calls to be fraudulent in certain regions of the country. Considering the disastrous impact such calls can have on the citizens in the times of emergency, it only makes much sense to identify such fraudulent calls as early as possible and handle them gracefully, thereby also enabling the emergency services IT infrastructure to fight any sort of DoS attack.

To mitigate the above-described issues, we propose a blockchain-based extension to the EMYNOS platform to handle such scenarios smoothly and efficiently. Every time after an emergency call ends, the PSAP operator could provide a short feedback for the caller, on a discrete scale e.g. on a scale of 5 , where 1 means a definite hoax call and 5 means an emergency or alternatively even a simpler feedback mechanism that accepts only binary input to mark the authenticity of the call. The feedback would implicitly contain other anonymised metadata associated with the call such as the telephone number (masked), time, location data etc. The feedback data could be stored on the blockchain and processed on-chain within a smart contract, to generate various metrics based on simple processing logic. Alternatively, the data could also be processed in batches on regular intervals off-chain (externally) and then the processed decision metrics post-processing could be updated on the blockchain. Off-chain processing would also support much more complex data processing algorithms. Once the metrics are available on the blockchain, regardless of onchain or off-chain processing, the ESRP when it receives a REGISTER request, can fetch the metrics against certain parameters extracted from the request, refer the sequence diagram in Fig. 7 , then evaluate the incoming call based on the fetched metrics and then forward the INVITE message to the Callee (i.e. the PSAP call taker) as usual in case of a negative evaluation $^{\S}$ or in case of a positive evaluation redirect the call to the IVR system that reads out an automated message that is set up to handle such fraud calls. The sequence diagram in Fig. 7 , is proposed as an extension to the standardised call routing

¥ The definition to categorise emergency calls varies for every PSAP. The report states, false emergency calls may constitute of accidental pocket calls, accidental dials and calls made because of misjudged situations etc. And, a deliberate call to the emergency services to inform the PSAP of an inexistent emergency would classify as a hoax or a fraud call.

$\S$ Positive evaluation means detection of a fraud call and vice-versa. procedure as in Figure 5.2 in [22], per the $\mathrm{M} / 493$ standardisation mandate [11].

To realise this idea, it would necessitate setting up a dedicated permissioned blockchain network at the EU level. Access to the blockchain would be granted to all involved PSAPs. Because of the sharing and availability of the data across the network, the fraudulent emergency calls could be more efficiently handled based on the caller's history across multiple locations and their prior interactions with various PSAPs from within the EU. Using blockchain would ensure traceability of all the stored data and would facilitate the immutable logging ${ }^{* *}$ of the data without a dedicated centralised instance in a P2P manner. Based on the blockchain based logs sophisticated autonomic and AI based algorithms can be triggered as to perform the required analysis and issue a corresponding reaction. Similar autonomic approaches were proposed in corresponding works and architectures from the domain of network and systems management [14][27][26].

To support the proposed solution, we would require extending some of the existing components in the current EMYNOS architecture. The Linphone client used by the PSAP call taker needs to support the feedback mechanism while the ESRP component would require to connect to the blockchain network to query the metrics on-demand and process the

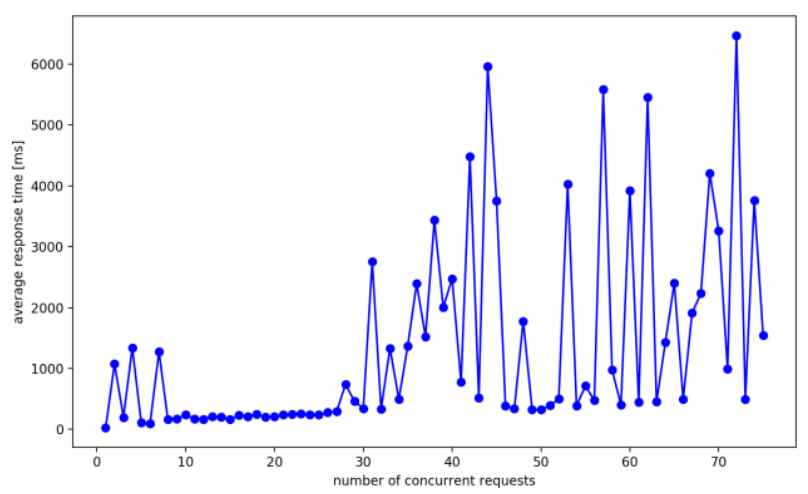

Fig. 8: Average response time per HTTP GET request to read data first from the blockchain and other associated data subsequently from the database incoming calls accordingly.

The Average Time per call and the Average Latency values as depicted in Fig. 3 through Fig. 5 seem very promising. So, introducing a little more complexity to the architecture with the fraud detection system to ensure a more reliable emergency support platform is definitely worth the try to test it as a concept. As per our experience, read latency for pre-processed data from the blockchain seems low, refer Fig. 8. The figure is an output for load tests where the requests read data first from a private Ethereum blockchain network (running in the data centre of Fraunhofer FOKUS), based on which more data is gathered from a traditional database and processed before sending out the response. The spikes observed in the graph could probably be attributed to database bottlenecks and/or the OS maintenance activities on the server and the virtual containers running the blockchain and database services because of the test load. We believe, the latencies can be significantly lowered with additional code and infrastructure optimisations, which was not the primary focus for the project. Hence, taking into consideration all the information we possess

** Assuming that the majority of the miners in the network are honest and that the mining power is not concentrated but shared across all the involved members maintaining the network. 
about the latency values, the performance of the resulting proposed solution should be worth the consideration in the scope of NG112 VoIP based emergency communications.

\section{Summary \& Outlook}

The current paper presents our activities related to the performance testing of VoIP emergency services. Given the advantages provided by NG112 IP based emergency communications, it is a lucrative step to initiate a transition from legacy PSTN architectures to IP based emergency architectures. These advantages include the potential integration of different types of communication such as voice, video, and real-time text for providing advanced options for handling an emergency. Furthermore, it is possible to integrate data from social networks as well as sensor data, e.g. environmental sensors or health sensors monitoring the human body. Despite all these advantages, the introduction of NG112 comes with a couple of pitfalls. Many experts doubt the actual performance (in terms of computational time and communications) of an IP based emergency system. Hence, there is a need for methods, tools and testbeds that will support the evaluation of emergency services over IP. Therefore, in the current paper, we present the EMYNOS testbed for emulating emergency services involving the whole variety of components (DHCP servers, LOST and HELD servers, sensor network integration, ESRP, PSAP, etc.) according to various standards such as the European M.493 and the US Next Generation 911. Based on a described experimental setup, a large number of measurements were conducted, which are correspondingly presented and show that VoIP emergency services stand a chance to perform reasonably and substitute legacy PSTN systems thereby increasing the richness of provided data and improving the chances to save human life in general. Furthermore, the utilisation of IP as an underlying substrate allows to integrate with novel service platforms such as blockchain and to implement various use cases such as the DoS fraudulent call detection scenario proposed in this paper. The belonging concepts for the DoS detection use case were elaborated and some performance measurements relating to the Ethereum blockchain technological platform presented in the current paper.

In general, we can conclude that the VoIP system is better in terms of functional features (sensor communication, RTT, Video, location-based aspects ...). The big question is whether it can reasonably perform in an environment where different types of IP traffic runs over the same network. This question is what the current paper tries to answer and give indications and a reasonable feeling regarding the reliability and performance of VoIP emergency communication. Based on the obtained measurements, we can confirm that VoIP emergency communication has the potential to play a key role as a future critical infrastructure.

For future work, we plan the increased integration of sensor devices from the domain of e-health and body networks. Furthermore, the interplay between NG112 and emerging IoT architectures will be investigated and corresponding performance measurements conducted. Thereby, the methodology proposed in this paper will further evolve and will give the possibility to explore the characteristics of these emerging networks and make appropriate design decisions.

\section{References}

[1] The EMYNOS project, Link. www.emynos.eu, as of date 09.01.2019

[2] Y. Rebahi, K. T. Chiu, N. Tcholtchev, S. Hohberg, E. Pallis, E. Markakis, "Towards a Next Generation 112 Testbed: The EMYNOS ESInet", to appear in the Elsevier International Journal of Critical Infrastructure Protection

[3] Next Generation 112 Long Term Definition, Link: https://de.scribd.com/document/89835743/EenaNg112-Ltd-v1-0-Final, as of date 27.02.2019

[4] Fraunhofer FOKUS https://www.fokus.fraunhofer de/en/sqc/technologi es/emytest, as of date 09.01.2019

[5] ETSI NG112 Plugtest \#2, Link: http://www.etsi.org/news-events/events/1110ng112-2, as of date 09.01.2019

[6] M. Manso et al., "The Application of Telematics and Smart Devices in Emergencies: Use Cases in Next Generation Emergency Services," 2016 IEEE First International Conference on Internet-of-Things Design and Implementation (IOTDI), Berlin, 2016, pp. 289-292. https://doi.org/10.1109/loTDI.2015.21

[7] E. Sdongos, A. Bolovinou, M. Tsogas, A. Amditis, B. Guerra and M. Manso, "Next generation automated emergency calls - Specifying next generation ecall \& sensor-enabled emergency services," 2017 14th IEEE Annual Consumer Communications \& Networking Conference (CCNC), Las Vegas, NV, 2017, pp. 1-6. https://doi.org/10.1109/CCNC.2017.8015368

[8] E. K. Markakis, A. Lykourgiotis, I. Politis, A. Dagiuklas, Y. Rebahi and E. Pallis, "EMYNOS: Next Generation Emergency Communication," in IEEE Communications Magazine, vol. 55, no. 1, pp. 139145, January 2017. https://doi.org/10.1109/MCOM.2017.1600284CM

[9] Evangelos K. Markakis, Ilias Politis, Asimakis Lykourgiotis, Yacine Rebahi, George Mastorakis, Constandinos X. Mavromoustakis, Evangelos Pallis, "Efficient Next Generation Emergency Communications over Multi-Access Edge Computing", Communications Magazine IEEE, vol. 55, pp. 92-97, 2017, ISSN 0163-6804. https://doi.org/10.1109/MCOM.2017.1700345

[10] R. Barnes and B. Rosen, "911 for the 21st Century," in IEEE Spectrum, vol. 51, no. 4, pp. 58-64, April 2014. https://doi.org/10.1109/MSPEC.2014.6776307

[11] $M / 493$, Standardization Mandate to the European Standards Organizations (ESO) in support of the Location Enhanced Emergency Call Service, Accepted by ETSI at Board\#84 on 7 November 2011

[12] NENA Standards \& Other Documents, NENA Functional and Interface Standards for Next Generation 9-1-1, online available: 
https://www.nena.org/page/standards, as of date 09.01.2019

[13] H2020-NEXES NEXt generation Emergency Services, http://nexes.eu/, as of date 09.01.2019

[14] N. Tcholtchev and R. Chaparadza, "Autonomic FaultManagement and resilience from the perspective of the network operation personnel," 2010 IEEE Globecom Workshops, Miami, FL, 2010, pp. 469-474. doi: $\quad$ 10.1109/GLOCOMW.2010.5700364 https://doi.org/10.1109/GLOCOMW.2010.5700364

[15] FP7- EmerGent, http://www.fp7-emergent.eu/, as of date 09.01.2019

[16] Help-112, http://eena.org/pages/help-112, as of date 09.01.2019

[17] Reach-112,

http://www.omnitor.se/en/projects/reach-112/, as of date 09.01.2019

[18] ITU-T Recommendation: Q.700 : Introduction to CCITT Signalling System No. 7, online: http://www.itu.int/rec/T-REC-Q.700/en, as of date 09.01.2019

[19] EU Commission, Digital Single Market Policy, eCall: Time saved = lives saved, online: https://ec.europa.eu/digital-single-market/ecalltime-saved-lives-saved, as of date 09.01.2019

[20] EENA Operations Document: False Emergency Calls [Annex - Statistics and definitions], https://eena.org/wpcontent/uploads/2018/11/False-EmergencyCalls.pdf, as of date 07.08.2019

[21] Fraudulent Calls and Hanging Up: PSAPs in the News, online: https://www.kovacorp.com/fraudulent-callsand-hanging-up-psaps-in-the-news/, as of date 21.08.2019
[22] ETSI ES 203178 v1.1.1, Functional architecture to support European requirements on emergency caller location determination and transport, https://www.etsi.org/deliver/etsi_es/203100_20319 9/203178/01.01.01_60/es_203178v010101p.pdf, as of date 22.08.2019

[23] Rebahi, Y., Sher, M., \& Magedanz, T. (2008, March). Detecting flooding attacks against IP Multimedia Subsystem (IMS) networks. In 2008 IEEE/ACS International Conference on Computer Systems and Applications (pp. 848-851). IEEE. https://doi.org/10.1109/AICCSA.2008.4493627

[24] Onofrei, A. A., Rebahi, Y., Magedanz, T., \& Institute, F. F. (2010). Preventing distributed denial-of-service attacks on the IMS emergency services support through adaptive firewall pinholing. arXiv preprint arXiv:1003.5429.

[25] Rebahi, Y., Nassar, M., Magedanz, T., \& Festor, O. (2011). A survey on fraud and service misuse in voice over IP (VoIP) networks. Information Security Technical Report, 16(1), 12-19. https://doi.org/10.1016/j.istr.2010.10.012

[26] R. Chaparadza, et.al.:, "Standardization of resilience \& survivability, and autonomic fault-management, in evolving and future networks: An ongoing initiative recently launched in ETSI," 2013 9th International Conference on the Design of Reliable Communication Networks (DRCN), Budapest, 2013, pp. 331-341.

[27] Timotheus Kastrinogiannis, et.al.: Addressing Stability in Future Autonomic Networking. MONAMI 2010: 50-61 https://doi.org/10.1007/9783-642-21444-8_5

[28] Wu, Woody. "Processing of emergency calls in wireless communications system with fraud protection." U.S. Patent 6,038,440, issued March 14, 2000. 